\title{
Position-specific carbon isotope analysis of amino acids by Orbitrap mass spectrometry to detect signatures of environmental and physiological change
}

\author{
ELISE B WILKES ${ }^{1}$, ALEX SESSIONS ${ }^{1}$, BRIAN \\ SCHUBERT $^{2}$, SARAH ZEICHNER ${ }^{1}$, GABRIELLA M. \\ WEISS $^{1,3}$ AND JOHN EILER ${ }^{1}$ \\ ${ }^{2}$ School of Geosciences, University of Louisiana at Lafayette \\ ${ }^{3}$ Pennsylvania State University \\ Presenting Author: ebwilkes@caltech.edu
}

${ }^{1}$ Caltech

Position-specific isotope patterns within amino acids hold potential as detailed tracers of metabolic fluxes and their interplay with the environment. Isotopic fractionation occurs at particular atomic positions within molecules [1], but this spatial information is lost (i.e., averaged) in conventional isotope ratio mass spectrometry. Organic geochemists may wish to exploit natural isotopic variations within biomolecules to develop novel proxies for environmental and physiological processes. However, isotope ordering within amino acids from natural samples has remained largely inaccessible to date due to methodological limitations. Intramolecular ${ }^{13} \mathrm{C} /{ }^{12} \mathrm{C}$ variations have been characterized in amino acids by a variety of techniques [2-6], but primarily in pure, commercially available standards, or in samples expected to have very large isotopic contrasts (e.g., meteoritic alanine)-reflecting challenges associated with method sensitivities or complex sample matrices. Here, we will present a method for the position-specific isotope analysis of amino acids by an Orbitrap ${ }^{\mathrm{TM}}$ mass analyzer coupled to a gas chromatograph, using data from plant samples and serine standards as case studies. This work represents some of the first Orbitrap-based measurements of position-specific isotope contrasts in amino acids isolated from a biological matrix. This approach enables on-line isolation of serine from a mixture of amino acids, as well as characterization of $\delta^{13} \mathrm{C}$ values for each carbon position in serine. Data from well-characterized plants [7] and standards will be used to illustrate method validation and to propose a proxy for photorespiratory fluxes in the environment. Finally, we will discuss prospects for generalizing these approaches to other amino acids, Orbitrap platforms, and sample types relevant to the organic geochemistry community.

[1] Hayes (2001) Rev. Mineral. Geochem. 43, 225-277. [2] Abelson \& Hoering (1961) Proc. Natl. Acad. Sci. 47, 623-632. [3] Romek, Krzeminska, Remaud, Julien, Paneth \& Robins (2017) Arch. Biochem. Biophys. 635, 60-65. [4] Fry, Carter, Yamada \& Juchelka (2018) Rapid Commun. Mass Spectrom. 32, 992-1000. [5] Neubauer, Sweredoski, Moradian, Newman, Robins \& Eiler (2018) Int. J. Mass Spectrom. 434, 276-286. [6] Chimiak, Elsila, Dallas, Dworkin, Aponte, Sessions \& Eiler (2021) Geochim. Cosmochim. Acta 292, 188-202. [7] Schubert \& Jahren (2018) Earth Sci. Rev. 177, 637-642. 